\title{
Accuracy of Parabolic Equation Method for Modeling LF Electromagnetic Wave Propagation
}

\author{
Zaojun Zhou ${ }^{1}$, Dandan Wang ${ }^{2}$, Yurong $\mathrm{Pu}^{2}$ and Xiaoli $\mathrm{Xi}^{2, *}$ \\ ${ }^{1}$ The Chinese People’s liberation army navy 91821 troops, Raoping, 515729, China \\ ${ }^{2} X i$ 'an University of Technology, Xi'an, 710048, China \\ ${ }^{*}$ Corresponding author
}

\begin{abstract}
The parabolic equation (PE) method, based on the split-step Fourier transform (SSFT) algorithm, has been used to model low-frequency (LF) electromagnetic (EM) wave propagation owing to its efficiency. However, the PE method is derived under the paraxial approximation so that its accuracy is guaranteed only for limited propagation angles from the paraxial direction. Most importantly, the PE method models only one-way forward EM propagation and neglects the backward one, which may become significant in the presence of steep terrain. Therefore, it is essential to properly estimate the accuracy of both narrow-angle (NA) PE (NAPE) and wide-angle (WA) PE (WAPE) methods for LF EM wave propagation prediction over irregular terrain. Since the finite-difference time-domain (FDTD) method has been proven to be the most precise method, in this paper, both the NAPE and WAPE methods are calibrated against finitedifference time-domain (FDTD) method over various paths.
\end{abstract}

Keywords-parabolic equation (PE) method; low-frequency (LF) electromagnetic waves propagation; finite-difference time-domain (FDTD)

\section{INTRODUCTION}

Low-frequency (LF) electromagnetic (EM) waves have played an important role for positioning, navigation and timing (PNT) and military communication systems. It is crucial to accurately model the LF EM wave propagation characteristics including the path-loss prediction over various propagation paths.

Recently, the numerical methods including finite-difference time-domain (FDTD) [1]-[4] and parabolic equation (PE) method [5]-[9] are employed to predict LF EM wave propagation over irregular terrain. The FDTD method has been shown to be the most precise one for arbitrary terrain, but its time step size is limited by the well-known Courant-FriedrichLevy (CFL) stability condition, which leads to large total simulation time [1]. The PE method is efficient for the propagation of LF EM waves over long distances, whereas its accuracy is restricted at certain angles due to the paraxial approximation [10], [11]. Most importantly, the PE method characterizes only forward-propagating waves, and neglects backward-propagating waves, which may produce significant errors over steep terrain [12]. More recently, the PE method is examined in short ranges against the FDTD method to obtain fast and accurate numerical simulation [11]. Hence, it is essential to appropriately estimate how accuracy the PE method can achieve in the presence of obstacles before application.

In this paper, the accuracy of the NAPE and WAPE methods for predicting LF EM wave propagation over various paths is calibrated against FDTD method. The calculated results show an assessment of both the NAPE and WAPE methods for further application.

\section{FORMULATION}

\section{A. PE Method}

Formulated in a cylindrical coordinate system, the NAPE and WAPE, respectively, are written as (with time dependence $\exp (-i \omega t))[10]$

$$
\frac{\partial u(\rho, z)}{\partial \rho}=\frac{i k_{0}}{2}\left[\frac{1}{k_{0}^{2}} \frac{\partial^{2}}{\partial z^{2}}+n^{2}-1\right] u(\rho, z)
$$

$\frac{\partial u(\rho, z)}{\partial \rho}=i k_{0}\left[\sqrt{1+\frac{1}{k_{0}^{2}} \frac{\partial^{2}}{\partial z^{2}}}-1\right] u(\rho, z)+i k_{0}(n-1) u(\rho, z)$

where $k_{0}$ is the wave number in air, $n$ is the refractive index of air, and $u$ is an auxiliary field defined as

$$
u(\rho, z)=H_{\varphi}(u, v) e^{-i k_{0} \rho} \sqrt{k_{0} \rho}
$$

The split-step PE (SSPE) solution for narrow and wide angles are shown in [10]

$$
\begin{aligned}
& u(\rho+\Delta \rho, z)= \exp \left[\frac{i k_{0}}{2}\left(n^{2}-1\right) \Delta \rho\right] \\
& \times \mathfrak{I}^{-1}\left\{\exp \left[\frac{-i p^{2}}{2 k_{0}} \Delta \rho\right] \mathfrak{I}\{u(\rho, z)\}\right\} \\
& u(\rho+\Delta \rho, z)= \exp \left[i k_{0}(n-1) \Delta \rho\right] \\
& \times \mathfrak{I}^{-1}\left\{\exp \left[i\left[\sqrt{k_{0}-p^{2}}-k_{0}\right] \Delta \rho\right] \mathfrak{I}\{u(\rho, z)\}\right\}
\end{aligned}
$$

where $\mathfrak{I}$ and $\mathfrak{I}^{-1}$ are the forward and inverse discrete mixed Fourier transform [10]. The transform variable $p$ is defined as $p=k_{0} \sin \theta$, where $\theta$ is the angle relative to the horizontal. 


\section{B. FDTD Method}

To simply the problem, but without loss of generality, the $H_{\phi}$ component is taken as an example. In the same coordinate system, the $H_{\phi}$ can be expressed as [4]

$$
\frac{1}{\rho} \frac{\partial\left(\breve{\rho} H_{\phi}\right)}{\partial \rho}=\mathrm{i} \omega \varepsilon_{0} \varepsilon_{r} \frac{S_{\rho} \breve{\rho}}{\rho} \breve{E}_{z}
$$

where

$$
\begin{gathered}
\breve{E}_{z}=S_{z} E_{z} \\
\breve{\rho}=\rho_{0}+\int_{\rho_{0}}^{\rho} S_{\rho}(\xi) d \xi .
\end{gathered}
$$

$\rho_{0}$ is PML absorbing boundary interface. Here, the stretching factors $S_{z}$ and $S_{\rho}$ are given by [4]

$$
\begin{gathered}
S_{z}(z)=k_{z}+\frac{\sigma_{z}}{\mathrm{i} \omega \varepsilon_{0}} \\
S_{\rho}(\rho)=k_{\rho}+\frac{\sigma_{\rho}}{\mathrm{i} \omega \varepsilon_{0}} .
\end{gathered}
$$

$k_{z}, k_{\rho}, \sigma_{z}$, and $\sigma_{\rho}$ in (9)-(10) are parameters of the PML.

\section{NUMERICAL RESULTS}

In this section, we provide some numerical results to evaluate the accuracy of the NAPE and WAPE methods against the FDTD method over irregular terrain, respectively. For all PE calculations, the range step $\Delta \rho$ and height step $\Delta z$, respectively, are $200 \mathrm{~m}$ and $100 \mathrm{~m}$. The computational domain of interest has a height of $35 \mathrm{~km}$ and range of $100 \mathrm{~km}$. The initial field is calculated by flat-earth formula at range $10 \mathrm{~km}$ [9]. The vertical electrical dipole is located at the coordinate origin $O$ at $100 \mathrm{kHz}$. The electric parameters for island are $\varepsilon_{r}=13$ and $\sigma=3 \times 10^{-3} \mathrm{~S} / \mathrm{m}$. The difference of field strength is calculated by

$$
\operatorname{error}(\mathrm{dB})=\left|H_{\varphi_{-} \mathrm{FDTD}}(\mathrm{dB})-H_{\varphi_{-} \mathrm{PE}}(\mathrm{dB})\right| .
$$

The criterion for the accurately simulated results is corresponding to the error less than $0.02 \mathrm{~dB}$.

First, the differences of field strengths between the NAPE and WAPE methods against the FDTD method are calculated over a flat propagation path. It is observed from the Fig. 1 that, based on the combination of the flat-earth formula and PE method mentioned in [9], the propagation angles of NAPE and WAPE are both extended about $10^{\circ}$. In addition, the error figure of the WAPE method is not smooth like the NAPE one. In other words, the path-loss prediction simulated by the WAPE method produces excess jitter along the range or height.

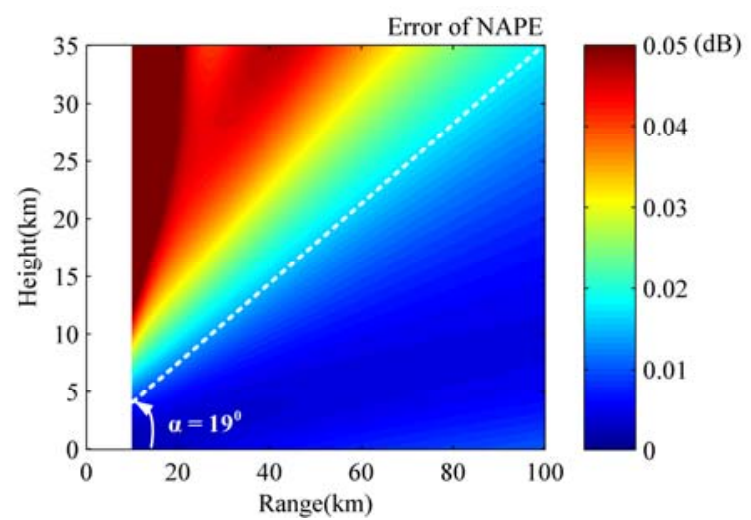

(a)

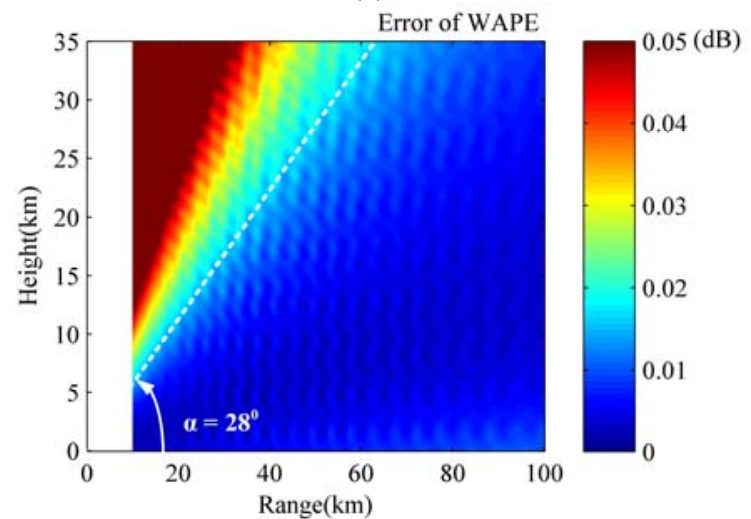

(b)

FIGURE I. ERRORS OF FIELD STRENGTHS BETWEEN (A) NAPE, (B) WAPE AGAINST FDTD METHODS ALONG FLAT EARTH PATH

The second example is intended to estimate the accuracy of NAPE and WAPE over a single Gaussian shaped mountain given by

$$
T(\rho)=H \exp \left[-9((\rho-50) / 2)^{2}\right]
$$

where $H$ is the maximum height of the mountain and the unit of $\rho$ need to be transferred to kilometer only. We tested three cases with different mountain heights of $0.5 \mathrm{~km}, 1.0 \mathrm{~km}$, and $1.5 \mathrm{~km}$, respectively. The calibration of NAPE and WAPE methods against FDTD method over irregular terrain is shown in Fig. 2. As observed in Fig.2, both the NAPE and WAPE methods agree fair well with the FDTD one when the mountain height is low. For the higher mountain, the errors of both the NAPE and WAPE methods increase in the front of the mountain due to the neglect of the backward-propagating waves. More importantly, it is crucial to find out that the WAPE method cannot provide the correct solution for the steeper mountains, whereas the NAPE method performs well compared with the FDTD one. 


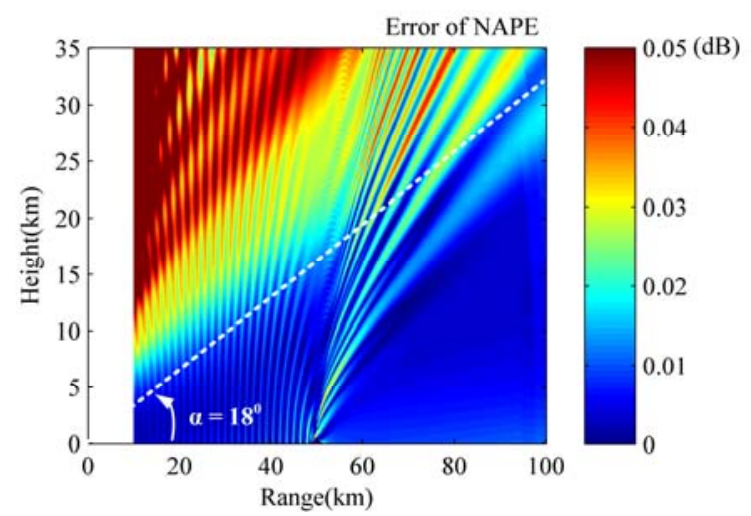

(a) Mountain height: $0.5 \mathrm{~km}$

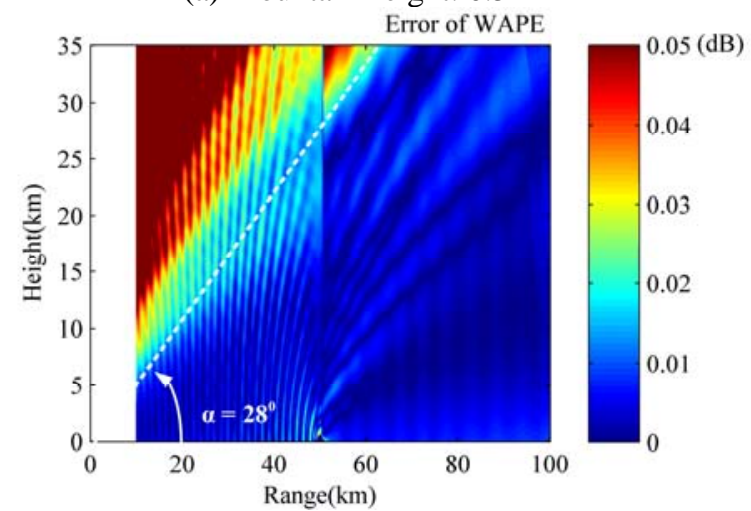

(b) Mountain height: $0.5 \mathrm{~km}$

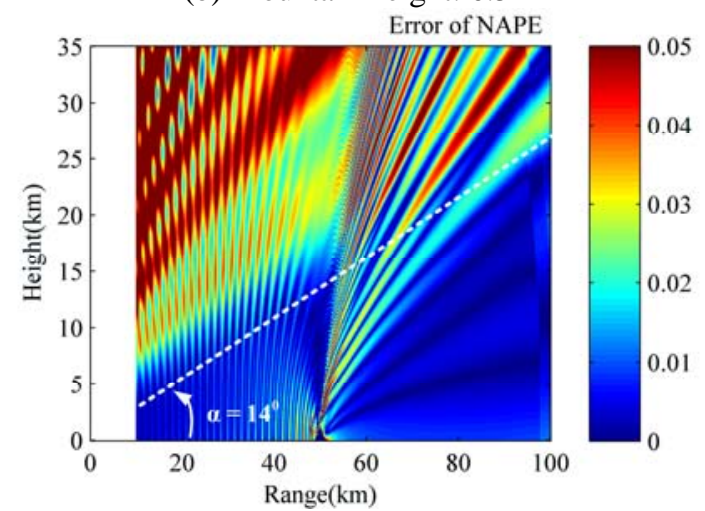

(c) Mountain height: $1.0 \mathrm{~km}$

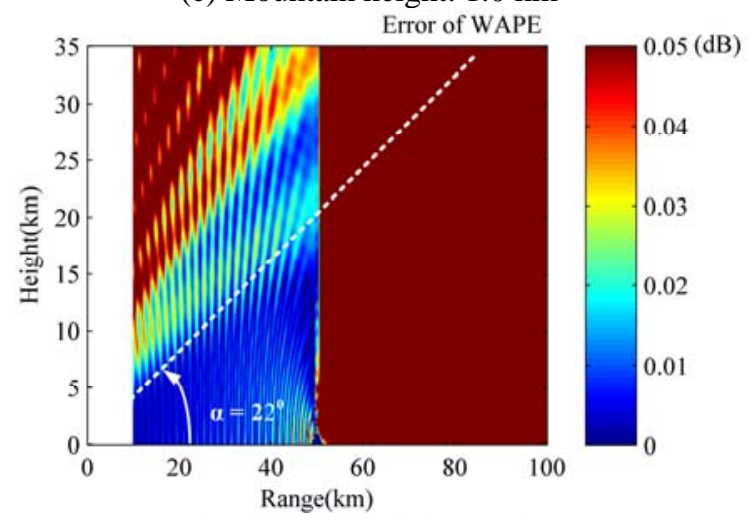

(d) Mountain height: $1.0 \mathrm{~km}$

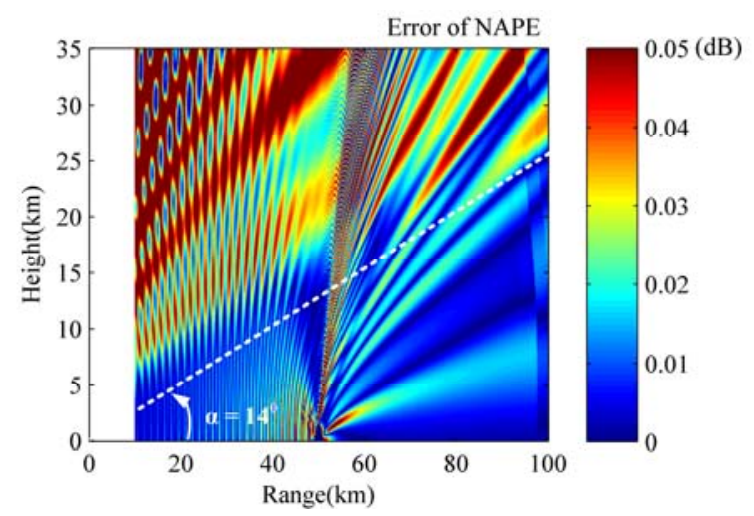

(e) Mountain height: $1.5 \mathrm{~km}$

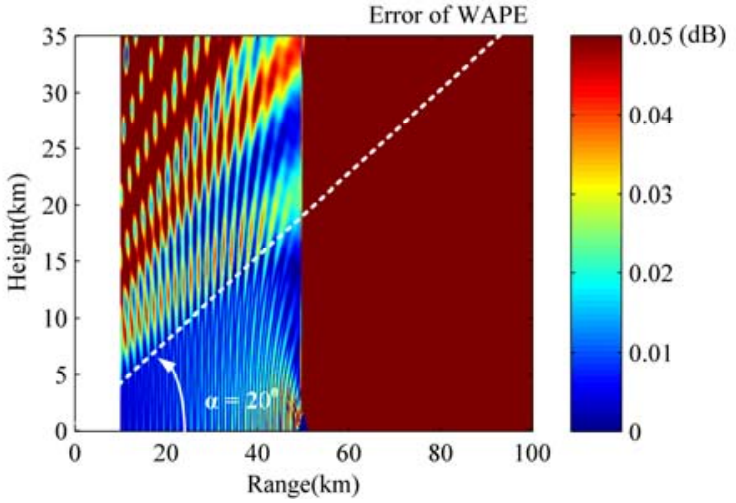

(f) Mountain height: $1.5 \mathrm{~km}$

FIGURE II. ERRORS OF FIELD STRENGTHS BETWEEN NAPE AND WAPE AGAINST FDTD METHODS ALONG DIFFERENT MOUNTAIN HEIGHTS

\section{CONCLUSION}

This paper examines the accuracy of NAPE and WAPE methods for modeling LF EM wave propagation over irregular terrain. The calibration is performed against the FDTD method. Numerical results show that the WAPE method has higher accuracy than the NAPE with large propagation angles for smooth terrain. For steeper terrain, the NAPE method matches the FDTD results well, whereas the WAPE method almost cannot work, but the strong effect of the backward-propagating waves is neglected due to the nature of one-way method itself for the both methods. The main conclusion of the paper is that the effect of the backward-propagating waves is significant for higher mountain, and hence needs to be taken into account with the two-way NAPE rather than the two-way WAPE for further study.

\section{REFERENCES}

[1] A. Taflove and S. C. Hagness, Computational Electrodynamics: The Finite-Difference Time-Domain Method, 3rd ed. Norwood, MA, USA: Artech House, 2005.

[2] L. Zhou, X. Xi, J. Zhang and Y. Pu, "A New Method for Loran-C ASF Calculation over Irregular Terrain," IEEE Transactions on Aerospace and Electronic Systems, vol. 49, no. 3, pp. 1738-1744, July 2013.

[3] L. Zhou, X. Xi, J. Liu and N. Yu, "LF Ground-Wave Propagation Over Irregular Terrain," IEEE Transactions on Antennas and Propagation, vol. 59, no. 4, pp. 1254-1260, April 2011. 
[4] L. Zhou, X. Xi, N. Yu and Y. Pu, "Modelling of LF ground-wave propagation at short distances based on 2-D cylindrical-coordinate FDTD method," in 2008 8th International Symposium on Antennas, Propagation and EM Theory, Kunming, 2008, pp. 855-858.

[5] L. Sevgi, F. Akleman and L. B. Felsen, "Groundwave propagation modeling: problem-matched analytical formulations and direct numerical techniques,” IEEE Antennas and Propagation Magazine, vol. 44, no. 1, pp. 55-75, Feb 2002.

[6] G. Apaydin and L. Sevgi, "Groundwave Propagation at Short Ranges and Accurate Source Modeling [Testing Ourselves]," in IEEE Antennas and Propagation Magazine, vol. 55, no. 3, pp. 244-262, June 2013.

[7] L. Sevgi, "Groundwave Modeling and Simulation Strategies and Path Loss Prediction Virtual Tools," IEEE Transactions on Antennas and Propagation, vol. 55, no. 6, pp. 1591-1598, June 2007.

[8] G. Apaydın, L. Sevgi, "MatLab-based Surface Wave Multi-Mixed Path Loss Calculator,” IEEE Antennas and Propagation Magazine, vol. 56, no. 3, pp.221-236, Jun. 2014

[9] D. D. Wang, X. L. Xi, Y. R. Pu, J. F. Liu and L. L. Zhou, "Parabolic Equation Method for Loran-C ASF Prediction Over Irregular Terrain," in IEEE Antennas and Wireless Propagation Letters, vol. 15, no. , pp. 734-737, 2016.

[10] M. Levy, Parabolic Equation Methods for Electromagnetic Wave Propagation, The Institution of Electrical Engineers, London, 2000.

[11] N. Omaki, Z. Q. Yun, and M. F. Iskander, "Accuracy of parabolic wave equation method in short propagation range," in Proc. 2012 IEEE Antennas and Propagation Society International Symposium, pp. 1-2, July 8-14, 2012.

[12] O. Ozgun, "Recursive Two-Way Parabolic Equation Approach for Modeling Terrain Effects in Tropospheric Propagation," IEEE Transactions on Antennas and Propagation, vol. 57, no. 9, pp. 27062714, Sept. 2009. 